\title{
Psychiatric Comorbidities in Children with Specific Learning Disorder-Mixed Type: A Cross-sectional Study
}

\author{
Anamika Sahu ${ }^{1}$ Vaibhav Patil ${ }^{2}$ Rajesh Sagar ${ }^{2}$ Rachna Bhargava ${ }^{3}$ \\ ${ }^{1}$ Department of Clinical Psychology, Central Institute of Psychiatry, \\ Ranchi, Jharkhand \\ 2Department of Psychiatry, All India Institute of Medical Sciences, \\ New Delhi, India \\ Address for correspondence Vaibhav Patil, MD, DNB, Department \\ of Psychiatry \& NDDTC Room No 4096, 4th floor, Academic Block, \\ All India Institute of Medical Sciences, New Delhi, 110029 \\ (e-mail: drvaibhavp317@gmail.com).
}

${ }^{3}$ Department of Psychiatry, National Drug Dependence Treatment Centre, All India Institute of Medical Sciences, New Delhi, India

| Neurosci Rural Pract 2019;10:617-622

\begin{abstract}
Background Specific learning disorder (SLD) is a neurodevelopmental condition which frequently exhibits with comorbidities of other disorders, including attention deficit hyperactivity disorder (ADHD), conduct disorder, anxiety, and depression. SLD with any comorbidity may affect the expression and severity of the SLD and may make its management difficult. Thus, the present cross-sectional study was planned to examine the psychiatric comorbidities among children with SLD.

Materials and Methods The sample consisted of 41 patients aged between 7 and 12 years with a diagnosis of SLD-mixed type. Clinical and psychological assessment included the following tests for behavioral, anxiety, mood, and interpersonal problems: child behavior checklist, Mini-international Neuropsychiatric Interview for Children and Adolescents (MINI-KID), and Conner's 3 Parent Short form-45.

Results The mean age of the participants was 9.8 years (standard deviation $[S D]=1.5)$. About $75.6 \%$ of participants were male, and their mean years of education was 5 years $(S D=1.5)$. Twenty-four percent of children had a history of delayed developmental milestones. Among comorbidities of SLD, association with attention

Keywords

- attention deficit hyperactivity disorder

- comorbidities

- specific learning disorders deficit disorder (ADD)/ADHD has been found to be significant along with difficulties in executive function, peer relation, and aggression.

Conclusion Children with SLD are likely to exhibit signs of ADHD/ADD and dysfunction in executive function, peer relation, and aggression. The management of comorbid conditions is recommended along with remediation of learning problem to overall educational and behavioral achievements and development of child.
\end{abstract}

\section{Introduction}

Specific learning disorder (SLD) is a general term that refers to a heterogeneous group of disorders manifested by significant difficulties in the acquisition and use of listening, speaking, reading, writing, reasoning, or mathematical abilities. ${ }^{1}$ It affects specific abilities in children with an otherwise normal academic development and with intact senses, normal intelligence, adequate motivation, and sociocultural opportunities. ${ }^{2}$ Globally, the prevalence of SLD has been estimated to be around 5 to $15 \%{ }^{3}$ while in India, it affects approximately 0.38 to $15.2 \%$ children. ${ }^{4}$ SLD begins in early childhood and continues across the lifespan with variable clinical expressions.

Children having SLD have been found to be suffering from various psychiatric comorbidities. Attention deficit 
hyperactivity disorder (ADHD) is the most common comorbid condition with SLD followed by anxiety disorder, mood disorder, and language disorder. ${ }^{5}$ Many children with SLD can experience low self-esteem, lack of confidence, low expectations for themselves, and poor peer relationship because of their poor learning skills. ${ }^{6}$ Studies from India have also reported various clinical problems associated with SLD, such as problem with developmental history, behavioral problems, neurotic traits, lower self-esteem, poor social competence, and high-state anxiety, that make management of SLD difficult. ${ }^{7-9}$

Sometimes learning problem contributes and/or triggers the maladaptive behaviors. Failure to achieve expected set targets or achieving lesser than desired outcomes often reflect feelings of isolation and a loss of interest in learning in these children. In addition, repeated failure or poor performance in spite of continuous efforts and practice can be responsible to develop a feeling of learned helplessness. Children may refuse to go to school and may complain somatic symptoms including frequent stomach aches, nausea, vomiting, and headache, to avoid performing on tests of academic skills which further hampers the educational development. In addition, some of them may indulge in disruptive behavior at home and/or classroom, for example, anger outburst and defiant behavior. ${ }^{10}$ Thus, early recognition and intervention for SLD with psychiatric comorbidities are required to improve outcome. However, only limited studies have paid attention to comorbid condition with SLD which have some methodological limitations. Hence, the present study was planned to examine the associated psychiatric comorbidities in children with SLD.

\section{Materials and Methods}

\section{Sample}

A total of 41children with SLD were participated in this cross-sectional study. Participants were recruited from the child guidance clinic run by psychiatry department of a tertiary care hospital in North India. All participants were diagnosed as cases of SLD by consultant psychiatrists. Further psychoeducational evaluation was done using SLD; comprehensive battery ${ }^{11}$ to identify the type of learning disorder in specific academic areas. All children were between 7 and 12 years of age, of either gender, and diagnosed with SLD-Mixed type as per International Classification of Diseases-10 (ICD-10) and had intelligence quotient more than $\geq 85$ (assessed on Malin's Intelligence Scale for Indian Children). ${ }^{12}$ Based on our clinical observation, we used children's age range between 7 and 12 years and SLD type as Mixed Type because maximum cases coming to the outpatient department of psychiatric had mixed type SLD with above-specific age range. Parents and children who were willing to participate were only included in the study. Participants were excluded if they had a known history of organic conditions, such as major neurological or medical disorders or impairment in hearing, vision, or speech, due to which learning difficulties could occur, or they refused to give informed consent for the study.

\section{Assessments}

Following scales and tests were administered as described below:

\section{Sociodemographic and Clinical Data Sheet for Children}

The datasheet was developed for the present study to gather details about sociodemographic and clinical profile of the children. Sociodemographic profiles included children's age, gender, education, religion, and residence and family type. A brief clinical history of children's developmental milestones, history of behavioral problem, major illness, and treatment history was obtained by means of datasheet.

\section{The Mini-international Neuropsychiatric Interview}

The Mini-international Neuropsychiatric Interview (MINI$\mathrm{KID})^{13}$ is a short-structured diagnostic interview, developed jointly by psychiatrists and clinicians in the United States and Europe, for Diagnostic and Statistical Manual of Mental Disorders, 4th Edition and ICD-10 psychiatric disorders. With an administration time of approximately 15 minutes, it was designed to meet the need for a short but accurate structured psychiatric interview for multicenter clinical trials and epidemiological studies. It screens 27 psychiatric disorders. Its sensitivity and specificity are 0.6 to 1.0 and 0.8 to 1.0 , respectively, for all diagnostic syndromes. The inter-rater reliability of the tool ranges from 0.64 to 1.00 for different diagnostic syndromes.

\section{Conners 3-Parent Short Form}

This test designed to identify ADHD and common comorbid problems, for example, learning problem, executive functions, defiance/aggression, and peer relation in children and adolescents with ages 6 to 18 years. Its administration time is 10 minutes. Reliability (test-retest reliability, 0.85 and internal consistency, 0.91 ) and validity of Conner's 3rd edition are high. ${ }^{14}$

\section{Child Behavior Checklist}

The child behavior checklist $(\mathrm{CBCL})^{15}$ is a device designed to assess social competence and behavior problems in children. It consists of 118 items related to behavior problems which are scored on a three-point scale ranging from not true to often true for the child. The CBCL allows for the circulation of raw scores and $t$-scores normed separately for girls and boys in eight different behavior domains as follows: withdrawn, somatic complaints, anxious/depressed, social problem, thought problems, attention problems, delinquent behavior, and aggressive behavior. These eight syndrome domains grouped into two higher order factors, internalizing (anxious/depressed, withdrawn, and somatic complaints scores) and externalizing (delinquent behavior and aggressive behavior). CBCL was chosen for the current study over Indian standardized version of CBCL, that is, childhood psychopathology measurement schedule ${ }^{16}$ to obtain detailed information about the child's different behavior problems, as well as internalizing, and externalizing problem. 


\section{Ethical Consideration}

Ethical approval to conduct the study was obtained from the institutional ethics committee. A written consent was collected from all parents before participating in the study.

\section{Procedure}

Participants meeting the ICD 10th Revision diagnosis of SLD-mixed type were screened and those who fulfilled the inclusion criteria were asked to participate in the study. Children and their parents were explained about the nature and purpose of the study. Written informed assent was obtained from the child and consent was taken by his/her caregivers or parents. After informed consent had been obtained, clinical and psychiatric interviews were conducted by a trained psychiatrist, while SLD and comorbidity assessments were administered by a trained clinical psychologist. Each interview session took approximately 2 hours to complete and individuals were offered periodic breaks during assessment.

\section{Analysis}

The data were analyzed using Statistical Package for Social Sciences software, Version 21.0 (SPSS, Chicago, Illinois, United States). Descriptive statistics was used to examine the data on demographic, clinical variables, and other psychopathology variables. It included frequency, percentage, means, and standard deviations (SDs).

\section{Results}

The demographic and clinical profile of the study participants are shown in - Table 1. Children with age ranged between 7 and 12 years were participated in the study (mean $\pm \mathrm{SD}=9.8 \pm 1.5$ ). Their classes ranged between $1 \mathrm{st}$ and 6th grades (mean $\pm \mathrm{SD}=5.0 \pm 1.5$ ). The sample was constituted primarily of boys (75.6\%). Majority of children were belonged to Hindu religion (90.2\%), nuclear family (58.5\%), and from middle socioeconomic strata (90.2\%). All the participants came from an urban background (100\%). Majority of the children were born through normal delivery (90\%). Only $17.1 \%$ of children had birth complication $(n=7)$ and out of which $4.9 \%$ of children had delayed cry $(n=2)$. Approximately, $24.4 \%$ of the participants had delayed milestones $(n=10)$.

- Fig. 1 depicts psychiatric comorbidity in children with SLD where $61 \%$ of children exhibited signs of attention deficit disorder (ADD; $n=14), \operatorname{ADHD}(n=9)$, social anxiety $(n=1)$, and both oppositional defiant disorder and ADD $(n=1)$. On Conners 3-Parent Short Form, substantial number of children had elevated scores on inattention $(n=28)$, hyperactivity $(n=11)$, executive function $(n=17)$, aggression $(n=23)$, and peer relation $(n=24)$. All children had higher score on learning problems (-Fig. 2). Similar to MINI-KID and Conners 3-Parent Short Form, children's scores obtained on all eight syndrome scales of the CBCL most frequently fell within the clinical range of scale that assessing symptoms of attention/hyperactivity $(n=21)$. Only one child had a pathological score on the externalizing scale $(t$-score $>64)$.
Table 1 Sociodemographic and clinical profile of children with specific learning disorder $(n=41)$

\begin{tabular}{|c|c|}
\hline Sociodemographic elements & Values \\
\hline Age $(y)^{\mathrm{a}}$ & $9.8 \pm 1.5$ \\
\hline Education $(y)^{a}$ & $5.0 \pm 1.5$ \\
\hline \multicolumn{2}{|l|}{ Gender $^{\mathrm{b}}$} \\
\hline Boys & $31(75.6)$ \\
\hline Girls & $10(24.4)$ \\
\hline \multicolumn{2}{|l|}{ Religion ${ }^{\mathrm{b}}$} \\
\hline Hindu & $37(90.2)$ \\
\hline Non-Hindu (Sikh and Christian) & $4(9.8)$ \\
\hline \multicolumn{2}{|l|}{ Family type ${ }^{b}$} \\
\hline Nuclear & $24(58.5)$ \\
\hline Joint & $17(41.5)$ \\
\hline \multicolumn{2}{|l|}{ Socioeconomic status $^{\mathrm{b}}$} \\
\hline Upper class & $4(9.8)$ \\
\hline Upper middle class & $29(70.7)$ \\
\hline Lower middle class & $8(19.5)$ \\
\hline \multicolumn{2}{|l|}{ Domicile $^{b}$} \\
\hline Urban & $41(100)$ \\
\hline \multicolumn{2}{|l|}{ Birth complication $^{\mathrm{b}}$} \\
\hline Present & $7(17.1)$ \\
\hline \multicolumn{2}{|l|}{ Delayed cryb } \\
\hline Present & $2(4.9)$ \\
\hline \multicolumn{2}{|l|}{ Delayed milestones ${ }^{b}$} \\
\hline Speech & $5(12.2)$ \\
\hline Walking & $3(7.3)$ \\
\hline Speech and walking & $2(4.9)$ \\
\hline \multicolumn{2}{|l|}{ Intelligence $^{\mathrm{a}}$} \\
\hline Full IQ & $105 \pm 9.0$ \\
\hline \multicolumn{2}{|l|}{ SLD } \\
\hline Mixed type & $41(100)$ \\
\hline
\end{tabular}

Abbreviations: IQ, intelligence quotient; SD, standard deviation; SLD, specific learning disorder.

${ }^{a}$ Mean \pm standard deviation. ${ }^{\mathrm{b}} n(\%)$.

\section{Discussion}

Associated other psychiatric disorder is very common with neurodevelopmental disorders, including SLD. It is important to have insight about comorbidity with SLD because an additional disorder may affect the expression and severity of the SLD. Learning-disabled children with comorbidity exhibit more severe neurocognitive impairment, negative academic experience, social outcomes, and lower treatment response as compared with children without other comorbid condition. ${ }^{5}$ Here, we present psychiatric comorbidity evaluation in children with SLD. 


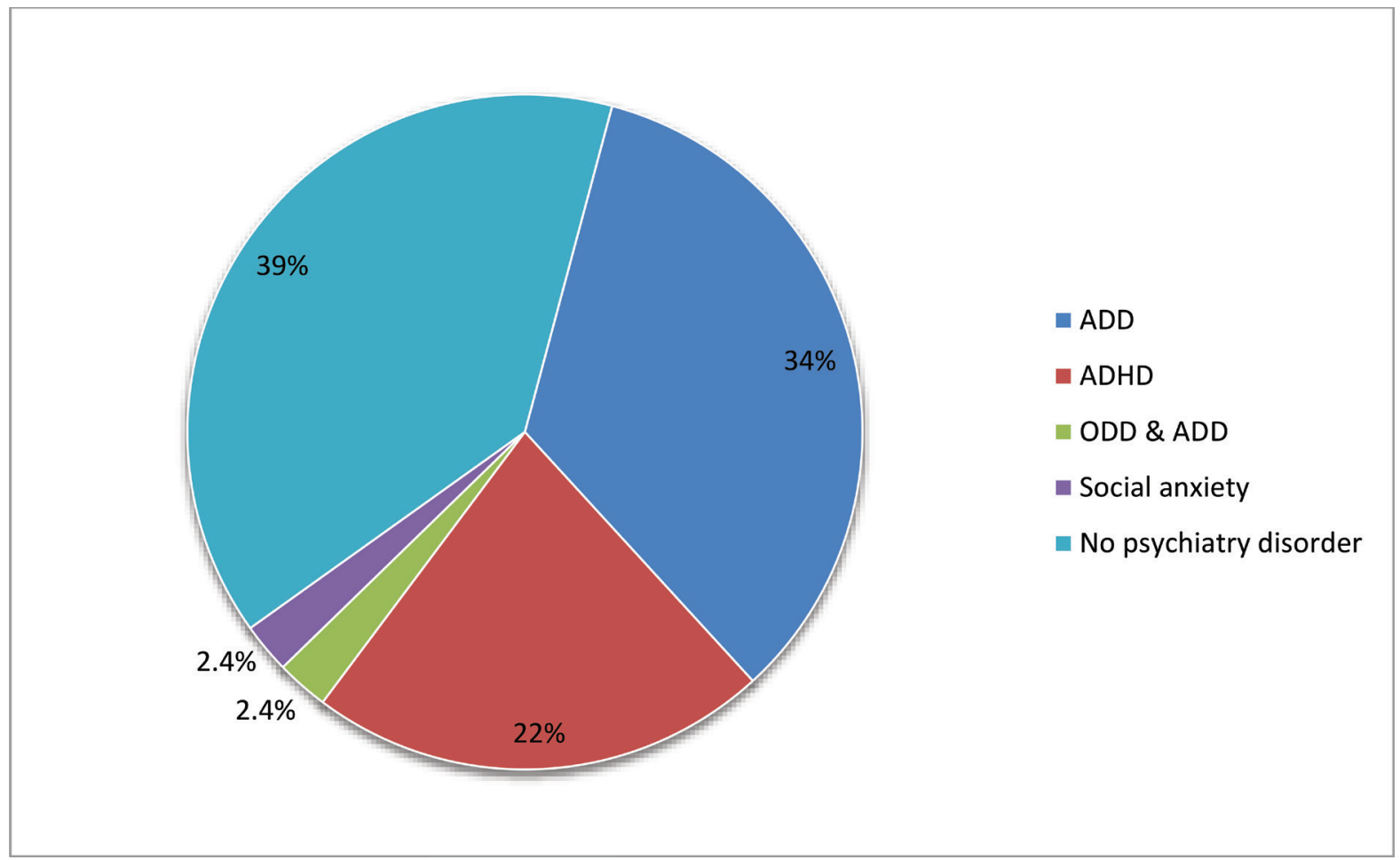

Fig. 1 Psychiatric comorbidity in children with specific learning disorder on MINI-KID. ADD, attention deficit disorder; ADHD, attention deficit hyperactivity disorder; MINI-KID, Mini-international Neuropsychiatric Interview; ODD, oppositional defiant disorder.

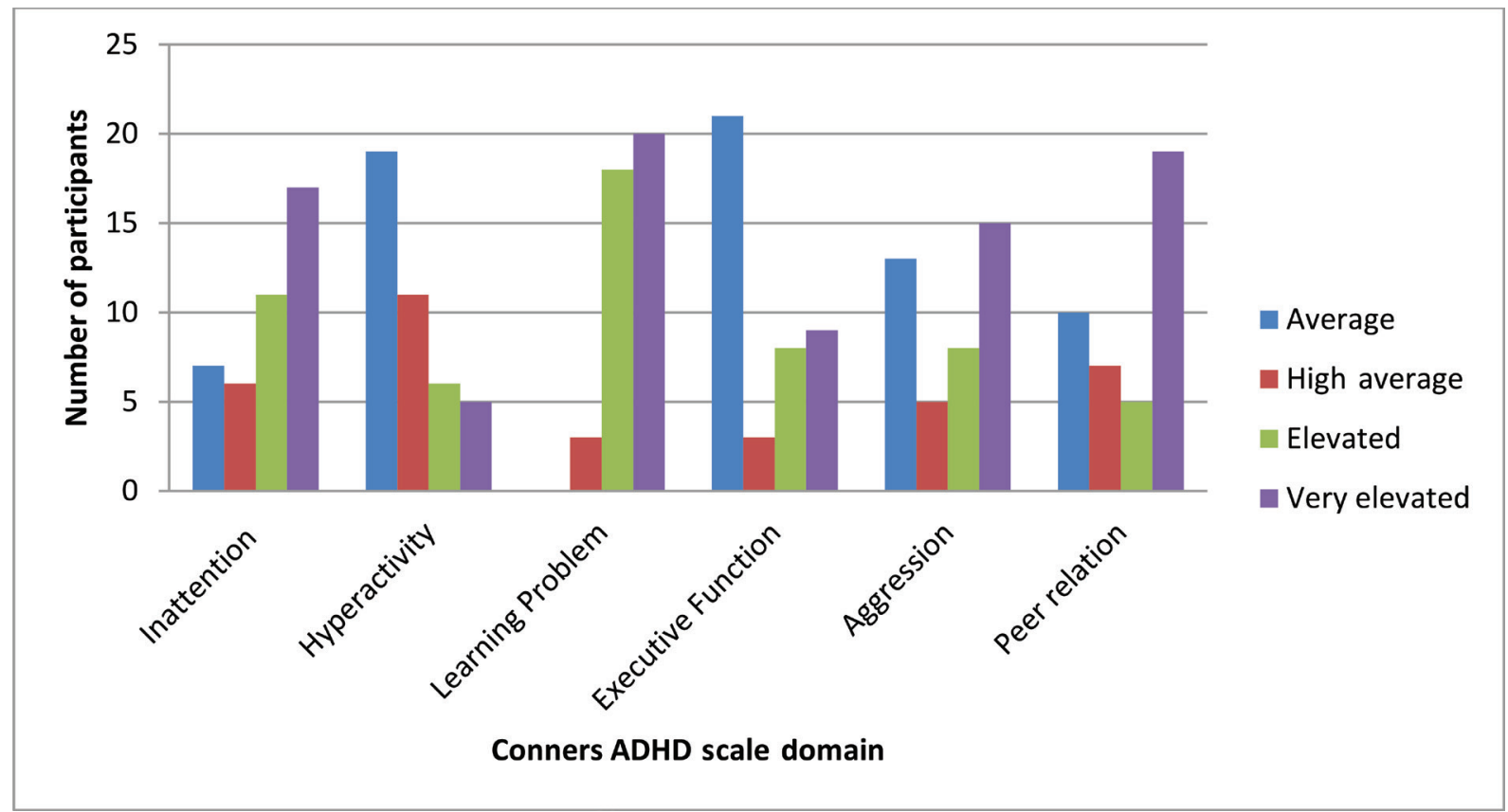

Fig. 2 Parents' responses on Conners attention deficit hyperactivity disorder scale.

The findings emerging from the current study indicate that ADD/ADHD is the most common psychiatric comorbidity with these children. This finding is in line with the previous studies that documented ADHD as the most common comorbid condition with SLD, $, 7,17$ as high as $45 \% .^{18}$ An Indian study has reported at least $10 \%$ of learning disabled children had clinical range of ADHD, ${ }^{19}$ while another study reported nearly $42 \%$ of ADHD. ${ }^{7}$ However, it was found that relation between dyslexia and inattention symptoms was stronger than to the hyperactive and impulsive symptoms of ADHD. ${ }^{20}$ In addition, only one child had social anxiety and another child had both oppositional defiant disorder and ADD. As compare with our study, higher prevalence of anxiety disorder and conduct disorder in SLD is reported by earlier studies. ${ }^{5,21,22}$ This could be due to small sample size and use of different measurement scales in the index study. 
On Conners 3-Parent Short Form, along with inattention, hyperactivity, and learning disorder, parents subjectively reported child's problems related to executive function, peer relationship, and aggressiveness. Researchers have also identified poor motor abilities, psychological process deficits, information processing problems, and attention memory as a core characteristic of SLD. ${ }^{23}$ Other studies have found deficits in memory, selective attention problem, visual memory, and verbal working memory. ${ }^{24,25}$ Literature is not conclusive regarding learning disability as a cause of aggressive or delinquent behavior; however, limited evidence suggests that reading disability may worsen preexisting aggressive behavior. ${ }^{26}$ Poor-peer relation and related emotional distress was also observed in our sample. Children had difficulty to form social connection, to getting along with other children, and to execute helpful behavior. This finding is consistent with previous reports mentioning that children with SLD had challenges in reading socioemotional cues, such as not knowing how to ask for help, being awkward in joining peer group, poor organization, planning, and impulse control. ${ }^{10}$

The findings of the study should be contextualized in terms of the strengths and limitations. The strength of the study includes the evaluation of psychiatric comorbidity in a homogeneous sample for first time, that is, SLD-Mixed type. In addition, we used measures that included self-reporting by parents and clinician's interview evaluations that were not followed by previous studies. A few important limitations need to be mentioned here. First, though more number of children with SLD included in the study; however, there is still a need for a larger sample to generalize the findings. Another limitation is the conduction of the study at tertiary care center that could not be representative of general population. Different results may be found from a community center and school setups.

\section{Conclusion}

This study extends our understanding of psychiatric comorbidities in the children with SLD-mixed type. There was a significant association of SLD with ADD/ADHD along with difficulties in executive function, aggression, and peer relation. The results add crucial points to the existing literature. However, much work is required with a more sophisticated study design. Hence, clinician should pay adequate attention for the evaluation of psychiatric comorbidities in these children. Moreover, our main aim should not be only to get insight about the psychiatric comorbidities and complication associated with SLD but to successfully identify child's needs that can then be integrated in the management plan which may facilitate effective and holistic improvement in child's educational and behavioral achievements.

\section{What Is Already Known?}

- Children with SLD aged between 7 and 16 years had psychiatric comorbidity with ADHD, anxiety disorder, mood disorder, and language disorder.

\section{What This Study Adds?}

- Children with SLD-mixed type aged between 7 and 12 years had most common comorbid condition of ADD/ ADHD along with dysfunction in executive function, peer relationship, and aggressive behavior.

\section{Funding}

None.

\section{Conflict of Interest}

None declared.

\section{References}

1 National joint committee on learning disabilities. Available from: http://www.ldonline.org/about/partners/njcld/archives. Accessed August 28, 2019

2 Shapiro BK, Gallico RP. Learning disabilities. Pediatr Clin North Am 1993;40(3):491-505

3 American Psychiatric Association, Diagnostic and Statistical Manual of Mental Disorders (DSM-5). 5th ed. Arlington: American Psychiatric Publishing; 2013

4 Bhargava R, Prakash S, Arun P, Epidemiology of specific learning disorder: Indian context. In: Sagar R, Pattanayak RD, eds. Specific Learning Disorder: Indian Scenario. New Delhi, India: Department of Science and Technology and AIIMS; 2014 133-54

5 Margari L, Buttiglione M, Craig F, et al. Neuropsychopathological comorbidities in learning disorders. BMC Neurol 2013;13:198

6 Cortiella C, Horowitz SH, The State of Learning Disabilities: Facts, Trends and Emerging Issues. New York, NY: National Center for Learning Disabilities; 2014

7 Bandla S, Mandadi GD, Bhogaraju A. Specific learning disabilities and psychiatric comorbidities in school children in South India. Indian J Psychol Med 2017;39(1):76-82

8 Kohli A, Malhotra S, Khehra N, Mohanty M. Deficits and clinical profile of the children with specific learning disability. Indian J Clin Psychol 2007;34:173-181

9 Sridevi G, George AG, Sriveni D, Rangaswami K. Learning disability and behaviour problems among school going children. J Disabil Stud 2015;1(1):4-9

10 Mehta M, Kapoor S, Learning difficulty: Academic and school related difficulties. In: Sagar R, Pattanayak RD, eds. Specific Learning Disorder: Indian Scenario. New Delhi, India: Department of Science and Technology (DST) and AIIMS; 2014 118-32

11 Mehta M, Sagar R, Specific Learning Disability Comprehensive Diagnostic Battery (SLD-CDB). New Delhi, India: Psychomatrix Publication; 2014

12 Malin AJ, Manual for Malin's Intelligence Scale for Indian Children (MISIC). Lucknow, India: Indian Psychological Corporation; 1969

13 Sheehan DV, Sheehan KH, Shytle RD, et al. Reliability and validity of the mini international neuropsychiatric interview for children and adolescents (MINI-KID) J Clin Psychiatry 2010;71(3):313-326

14 Conners CK, Pitkanen J, Rzepa SR, Conners. Manual. 3rd ed. New York, NY: Springer; 2008

15 Achenbach TM, Manual for the Child Behavior Checklist/4-18 and 1991 Profile. University of Vermont, Department of Psychiatry; 1991

16 Malhotra S, Varma VK, Verma SK, Malhotra A. Childhood psychopathology meausrement schedule: development and standardization. Indian J Psychiatry 1988;30(4):325-331 
17 Somale A, Kondekar SV, Rathi S, Iyer N. Neurodevelopmental comorbidity profile in specific learning disorders. Int J Contemp Pediatr 2016;3(2):355-361

18 DuPaul GJ, Gormley MJ, Laracy SD. Comorbidity of LD and ADHD: implications of DSM-5 for assessment and treatment. J Learn Disabil 2013;46(1):43-51

19 Gulati JK, Neha L, Jasvinder K. Attention deficit hyperactive disorder-as a determinant of learning disability. Asian J Home Sci 2015;10(1):196-202

20 Willcutt EG, Pennington BF. Psychiatric comorbidity in children and adolescents with reading disability. J Child Psychol Psychiatry 2000;41(8):1039-1048

21 Gallegos J, Langley A, Villegas D. Anxiety, depression, and coping skills among Mexican school children: A comparison of students with and without learning disabilities. Learn Disabil Q 2012;35(1):35
22 Thakkar AN, Karande S, Bala N, Sant H, Gogtay NJ, Sholapurwala R. Is anxiety more common in school students with newly diagnosed specific learning disabilities? A cross-sectional questionnaire-based study in Mumbai, Maharashtra, India. J Postgrad Med 2016;62(1):12-19

23 Mercer CD, Pullen PC, Students with Learning Disabilities. 7th ed. Upper Saddle River, NJ: Merrill-Prentice Hall; 2009

24 Agrawal S, Kar BR. Neuropsychological deficits in children with dyslexia. Indian J Clin Psychol 2007;34(2):164-172

25 Pattanayak RD, Sagar R, Choudhary V, Care giver perspectives based of focus group discussion: Parental burden, experiences and unmet needs. In: Sagar R, Pattanayak RD, eds. Specific Learning Disorder: Indian Scenario. New Delhi, India: Department of Science and Technology (DST) and AIIMS; 2014 265-77

26 Cornwall A, Bawden HN. Reading disabilities and aggression: a critical review. J Learn Disabil 1992;25(5):281-288 